\title{
Chronic widespread pain patients show disrupted cortical connectivity in default mode and salience networks, modulated by pain sensitivity
}

This article was published in the following Dove Press journal: Journal of Pain Research

\author{
Helene van Ettinger- \\ Veenstra $\mathbb{1}^{1,2}$ \\ Peter Lundberg $\mathbb{1}^{1,3,4}$ \\ Péter Alföldi (iD ${ }^{5}$ \\ Martin Södermark ${ }^{5}$ \\ Thomas Graven-Nielsen (1D) \\ Anna Sjörs (iD ${ }^{7}$ \\ Maria Engström (iD) ${ }^{1,4}$ \\ Björn Gerdle $\mathbb{D}^{1,5}$ \\ 'Center for Medical Image Science and \\ Visualization (CMIV), Linköping \\ University, Linköping, Sweden; ${ }^{2}$ Center \\ for Social and Affective Neuroscience \\ (CSAN), Department of Clinical and \\ Experimental Medicine, Linköping \\ University, Linköping, Sweden; \\ ${ }^{3}$ Department of Radiation Physics, \\ Linköping University, Linköping, Sweden \\ ${ }^{4}$ Department of Medical and Health \\ Sciences, Linköping University, Linköping, \\ Sweden; ${ }^{5}$ Pain and Rehabilitation Centre, \\ Department of Medical and Health \\ Sciences, Linköping University, Linköping, \\ Sweden; ${ }^{6}$ Center for Neuroplasticity and \\ Pain (CNAP), SMI, Aalborg University, \\ Aalborg, Denmark; ${ }^{7}$ Institute of Stress \\ Medicine, Region Västra Götaland and \\ Department of Clinical Neuroscience and \\ Rehabilitation, University of Gothenburg, \\ Gothenburg, Sweden
}

Correspondence: Helene van Ettinger-Veenstra

Center for Social and Affective Neuroscience (CSAN), Department of Clinical and Experimental Medicine, Linköping University, SE-58I 85 Linköping, Sweden

Tel +46I 3281000

Email helenevanettinger@gmail.com
Purpose: The remodeling of functional neuronal connectivity in chronic widespread pain (CWP) patients remains largely unexplored. This study aimed to investigate functional connectivity in CWP patients in brain networks related to chronic pain for changes related to pain sensitivity, psychological strain, and experienced pain.

Patients and methods: Functional connectivity strength of the default mode network $(\mathrm{DMN})$ and the salience network (SN) was assessed with functional magnetic resonance imaging. Between-group differences were investigated with an independent component analysis for altered connectivity within the whole DMN and SN. Then, changes in connectivity between nodes of the DMN and SN were investigated with the use of a seed-target analysis in relation to the covariates clinical pain intensity, pressure pain sensitivity, psychological strain, and as an effect of experienced experimental cuff-pressure pain.

Results: CWP patients showed decreased connectivity in the inferior posterior cingulate cortex (PCC) in the DMN and increased connectivity in the left anterior insula/superior temporal gyrus in the SN when compared to controls. Moreover, higher pain sensitivity in CWP when compared to controls was related to increased connectivity within the SN (between left and right insula) and between SN and DMN (between right insula and left lateral parietal cortex).

Conclusion: This study shows that connectivity within the DMN was decreased and connectivity within the SN was increased for CWP. Furthermore, we present a novel finding of interaction of pain sensitivity with SN and DMN-SN functional connectivity in CWP.

Keywords: functional connectivity, functional magnetic resonance imaging, fMRI, default node network, DMN, salience network

\section{Introduction}

Chronic widespread pain (CWP) and fibromyalgia (FM) are diagnoses that are associated with widespread pain, hypersensitivity, and increased psychological load. $^{1-3}$ In combination with the observation of altered central nervous system (CNS) processing in CWP, ${ }^{4,5}$ this explains the increasing interest in the association between large-scale neural networks and pain conditions. ${ }^{6,7}$ However, the reports of directionality of neuronal network changes related to chronic pain are showing a complex picture, and the research on CWP and the influence of clinical pain intensity, pain sensitivity, and psychological strain is currently only poorly understood. $^{8-13}$ Therefore, this study investigates neural network changes in CWP and their relation to clinical measures with the use of resting state functional magnetic resonance imaging (rs-fMRI). 
Functional connectivity changes in CNS related to CWP or FM can be viewed as the result of long-term remodeling upon chronic pain, but short-term changes have been observed as well. ${ }^{8-10}$ A stronger connectivity within the default mode network (DMN) was observed in $\mathrm{FM}^{8}{ }^{8}$ while, in contrast, this network was disrupted in chronic non-widespread pain patients. ${ }^{11,12}$ The DMN is associated with self-referential processing, rumination, and theory-of-mind processes that are not cued by external stimuli. ${ }^{14,15}$ Notably, the DMN is involved in modulation of pain perception through both structural and functional connections with antinociceptive and autonomic descending networks. ${ }^{16,17}$ The main nodes of the DMN are the medial prefrontal cortex (MPFC), the posterior cingulate cortex (PCC) and adjacent precuneus, and the lateral parietal cortex (LPC). ${ }^{18}$

Besides being characterized by DMN alterations, chronic pain patients have also repeatedly exhibited connectivity changes in insula and anterior cingulate cortex (ACC), as concluded in a recent review. ${ }^{5}$ FM patients have expressed increased connectivity between the insula and ACC when compared to healthy controls. ${ }^{10}$ The insula and ACC are parts of a descending pain modulatory system that facilitates or inhibits pain experiences through modulatory pain processing, but also through cognitive evaluation and behavioral choice making. ${ }^{19,20}$ Furthermore, these structures are involved in a neural signature for experimentally induced pain and painful states, independent of pain modality, location, or health condition. ${ }^{7,21}$ Bilateral anterior insular cortex (AIC) and dorsal ACC represent three major regions or nodes of the salience network $(\mathrm{SN}){ }^{22}$ The $\mathrm{SN}$ processes "bottom-up" aversive or other salient attentiondemanding stimuli or experiences such as pain, is in particular involved in the affective processing of pain, ${ }^{13,20,23}$ and shows also chronic pain related alterations. ${ }^{13}$

CWP and FM patients have been described as falling on a continuum between peripherally and centrally driven pain. $^{2}$ More widespread chronic pain is associated with more negative outcomes, ${ }^{24}$ and negative outcomes in CWP have been found to be affected by a complex interplay between pain aspects and psychological symptoms. ${ }^{25}$ Psychological distress-often reported by CWP patients $^{24-26}$ - has been associated with changes within the DMN and $\mathrm{SN}^{27-30}$ and is known to show interaction effects with neural correlates to chronic pain. ${ }^{31}$ The connectivity within the DMN and between the DMN and the insula and the ACC in chronic pain patients is affected by chronic pain duration as well as by evoked experimental pain and increased clinical pain. ${ }^{8,11-13,32,33}$ When modulating in the opposite direction; it has been shown that it is possible to reduce DMN functional connectivity as an effect of experimentally induced pain in healthy participants. ${ }^{32}$ Summing up; the literature strongly suggests that modulatory roles of pain aspects and psychological symptoms on functional connectivity - within as well as between the DMN and $\mathrm{SN}$ - are likely to occur in $\mathrm{CWP}^{34}$

DMN nodes normally show negative connectivity, sometimes described as anti-correlation, with SN nodes. ${ }^{14,20}$ However, the SN has been hypothesized to be "functionally damaged" in chronic pain patients as a consequence of a barrage of repeated painful input. ${ }^{13}$ Consistent with the previously discussed literature, a damaged SN may be an underlying cause for altered connectivity of DMN with insula or ACC. Most previous studies on CWP have focused on node-level interactions. However, in line with the emerging view of the brain as interactive network model, the current study aimed to explore the network-level changes related to CWP and the interaction of CWP and painful stimulation, pain sensitivity, or psychological distress.

At baseline in comparison to healthy controls, it was hypothesized that CWP would demonstrate (1) affected (reduced or increased) connectivity within and between the DMN and SN, and (2) altered connectivity related to the DMN and SN affected by clinical pain intensity, pain sensitivity, or psychological strain. Moreover, it was hypothesized that (3) the connectivity patterns observed at baseline were affected (reduced or increased) by experimental cuff-induced pain.

\section{Participants and methods Participants}

Thirty-nine female CWP patients and 40 female age-matched healthy control (HC) participants between 20 and 65 years were recruited. fMRI-data from $1 \mathrm{CWP}$ patient and $3 \mathrm{HCs}$ were of insufficient quality because of movement artefacts and brain coverage, and were excluded. Hence, 38 CWP patients and $37 \mathrm{HCs}$ were included in the analysis. The study was granted ethical clearances by Linköping University Ethics Committee (Dnr: 2010/235-31); all participants gave their written informed consent and the study was performed according with the Helsinki Declaration. All participants underwent a clinical examination, a questionnaire (Table 1), assessment of pain sensitivity, and an fMRI scanning session. 
Table I Age, anthropometric data, blood pressure, clinical pain intensity and psychological variables together with pain detection threshold for HCs and patients with CWP

\begin{tabular}{|c|c|c|c|c|c|}
\hline Group & HC $(n=37)$ & & CWP $(n=38)$ & & \\
\hline Variables & Mean & SD & Mean & SD & $p$-value \\
\hline Age (years) & 50.9 & 10.6 & 46.2 & 11.8 & 0.08 \\
\hline Weight (kg) & 66.7 & 10.1 & 68.2 & 11.7 & 0.57 \\
\hline Height (m) & 1.66 & 0.06 & 1.67 & 0.06 & 0.26 \\
\hline BMI $\left(\mathrm{kg} / \mathrm{m}^{2}\right)$ & 23.9 & 3.2 & 24.3 & 3.7 & 0.66 \\
\hline $\mathrm{SBP}(\mathrm{mmHg})$ & 123.6 & 14.9 & 125.4 & 15.4 & 0.62 \\
\hline $\mathrm{DBP}(\mathrm{mmHg})$ & 79.3 & 8.3 & 81.2 & 9.6 & 0.37 \\
\hline Number of tender points & - & - & 10.9 & 5.6 & - \\
\hline NRS-current (0-10) & 0 & 0 & 4.8 & 2.1 & $<0.001$ \\
\hline NRS-7 days $(0-10)$ & 0 & 0 & 5.9 & 1.9 & $<0.001$ \\
\hline Pain intensity-global (VAS; 0-100 mm) & 0.9 & 1.7 & 42.0 & 19.4 & $<0.001$ \\
\hline HADS-A & 2.3 & 2.4 & 6.6 & 4.6 & $<0.001$ \\
\hline HADS-D & 1.5 & 2.4 & 7.3 & 4.5 & $<0.001$ \\
\hline BDI & 3.3 & 5.1 & 17.2 & 12.6 & $<0.001$ \\
\hline BAI & 2.9 & 3.2 & 14.5 & 9.4 & $<0.001$ \\
\hline ASI & 6.6 & 5.6 & 16.0 & 12.3 & $<0.001$ \\
\hline PASS & 14.0 & 14.2 & 33.5 & 19.0 & $<0.001$ \\
\hline PCS & 8.1 & 8.5 & 16.5 & II.I & $<0.001$ \\
\hline Psychological strain & -0.5 & 0.4 & 0.5 & 0.9 & $<0.001$ \\
\hline Cuff pain detection threshold (PDT; $\mathrm{kPa}$ ) & 12.2 & 4.6 & 9.9 & 3.8 & 0.021 \\
\hline Cuff pain tolerance threshold (PTT; kPa) & 43.4 & 17.0 & 31.6 & 13.9 & 0.001 \\
\hline
\end{tabular}

Notes: The results of the statistical comparison ( $t$-test) are shown in the right column, significance (in bold) was set at a Bonferroni corrected $\alpha=0.05 / 19=0.003$ to correct for 19 correlations. NRS-current, current pain intensity according to a numerical rating scale (NRS); NRS-7 days, pain intensity according to NRS recent 7 days.

Abbreviations: HC, healthy control; CWP, chronic widespread pain; BMI, body mass index; SBP, systolic blood pressure; DBP, diastolic blood pressure; NRS, numerical rating scale; VAS, visual analogue scale; HADS-A, Hospital Anxiety and Depression Scale-Anxiety; HADS-D, Hospital Anxiety and Depression Scale-Depression; BDI, Beck Depression Inventory; BAI, Beck Anxiety Inventory; ASI, Anxiety Sensitivity Index; PASS, Pain Anxiety Symptoms Scale-20; PCS, Pain Catastrophizing Scale; PDT, pain detection threshold; PTT, pain tolerance threshold.

\section{Clinical examination}

The brief clinical examination was assessed primarily to check inclusion and exclusion criteria. Patients with CWP used various medications related to pain and psychological symptoms: paracetamol (37\%), nonsteroidal anti-inflammatory drugs (NSAID)/anti-inflammatory drugs (34\%), antidepressants $(29 \%)$, opioids $(26 \%)$, tricyclic antidepressants $(11 \%)$, anticonvulsants (5\%), triptans (5\%), muscle relaxants $(8 \%)$, and hypnotics for sleep (16\%). In the CWP group, 10 patients (26.3\%) were diagnosed with FM. HCs were pain-free according to their medical history. None of the HCs used medication related to pain or psychological symptoms. All participants were asked to refrain from pain medication during the experiment day. Inclusion criteria for CWP was widespread pain according to the American College of Rheumatology (ACR) criteria, ${ }^{35}$ with registered number of tender points, current pain intensity, and pain intensity during the last 7 days assessed on a numeric rating scale (NRS; range: 0-10, no pain to worst pain imaginable) during a clinical examination. Exclusion criteria for both groups were rheumatoid arthritis, metabolic disease, malignancy, cardiovascular disease, unregulated thyroid disease, lung disease, severe psychiatric conditions, pregnancy, difficulties understanding the Swedish language, and MR-incompatibility.

\section{Questionnaires}

Sociodemographic and anthropometric data

Age, self-reported weight and height were collected.

\section{Clinical pain intensity}

Pain intensity in nine different anatomical regions (neck, shoulders, arms, hands, upper back, low back, hips, knees and feet) was registered using an electronic visual analogue scale (VAS) where $0 \mathrm{~cm}$ was defined as "no pain" and $10 \mathrm{~cm}$ as "worst pain imaginable". The mean value of pain VAS scores across the nine anatomical regions was calculated (denoted as pain intensity-global).

\section{Psychological strain}

The two subscales from the Hospital Anxiety and Depression Scale of depressive (HADS-D) and anxiety 
(HADS-A) symptoms were used; range: 0-21. ${ }^{25,36}$ Symptoms of depression were also assessed with Beck Depression Inventory (BDI); range: $0-63,{ }^{24,37}$ and symptoms associated with anxiety and panic with Beck Anxiety Inventory (BAI); range: 0-63. ${ }^{38}$ Furthermore, Anxiety Sensitivity Index (ASI) to measure fear of sensations related to arousal; range: $0-64,{ }^{25,39}$ Pain Anxiety Symptoms Scale-20 (PASS-20) to measure fear and anxiety elated to pain; range: $0-100^{25,40}$ and Pain Catastrophizing Scale (PCS) regarding catastrophic thoughts related to pain; range: $0-52^{25,41}$ were used. For this study, the mean of the normalized scores (ie, mean $=0$ and $\mathrm{SD}=1$ for all subjects taken together) of these seven psychological variables (HADS-D, HADS-A, BDI, BAI, ASI, PASS-20, PCS) was calculated as a construct of psychological strain, ie, high values indicated high psychological strain. The internal consistency of the construct in this sample was excellent (Cronbach's $\alpha=[0.912]$ ).

\section{Assessment of pain sensitivity}

The pain sensitivity was assessed by cuff algometry to record the cuff pressure pain detection thresholds (PDT) and pain tolerance threshold (PTT) on leg and arm. ${ }^{42}$ The experimental setup consisted of a double chamber 13-cm wide tourniquet cuff (VBM Medizintechnik GmbH, Sulz, Germany) applied around the arm or the leg. A computer-controlled cuff algometer (NociTech, Denmark, and Aalborg University) was used to inflate the cuff by $1 \mathrm{kPa} /$ second to a maximum pressure limit of $100 \mathrm{kPa}$. The inflation could be aborted at any time by the participant or the experimenter. ${ }^{43}$ Simultaneously with the cuff stimulation, the participants scored the pain intensity on the pain VAS scale and aborted the stimulation when they could not tolerate further increase of cuff pressure. PDT was defined as the pressure equivalent to the moment of transition from strong to painful pressure (VAS $>0.1$ for the first time), PTT was defined as the equivalent of the moment of transition from painful to unbearable pressure. PDTs and PTTs were recorded twice at the right arm (ie, the cuff around the heads of biceps and triceps muscles) and right lower leg (ie, the cuff around the triceps surae). Mean PTT across trials and extremities was used as a measure of the general pain sensitivity; lower PTT corresponds to higher pain sensitivity.

\section{fMRI}

\section{Resting state $\mathrm{fMRI}$ paradigm}

Rs-fMRI was obtained directly before (baseline) and directly after experimental cuff-induced pain (post-pain) in sessions lasting 10 minutes, with eyes closed, on a Philips Ingenia 3T scanner with a SENSE head coil using a single-shot echo planar imaging (EPI) gradient echo sequence. MR parameters for the functional images were: repetition time $=2.2$ seconds, echo time $=35 \mathrm{~ms}$, flip angle $=77$ degrees, field-of-view $=240 \times 240 \times 105$, voxel size $=3 \times 3 \times 3 \mathrm{~mm}$ (no gap), number of slices $=35$. The alignment for the fMRI images was at the AC-PC line, with coverage of the whole cerebrum.

\section{Experimental pain stimulation}

Experimental pain after the baseline rs-fMRI scan was applied in two back-to-back cuff pain blocks each of about 7 minutes consisting of 20 seconds of pain interspersed with 20 seconds of rest. The experimental pain blocks consisted of one block of cuff pressure of $20 \mathrm{kPa}$ (corresponding to low pain intensity in healthy participants) $)^{42,44}$ and one block of individualized cuff pressure corresponding to a pain intensity of $7 \mathrm{~cm}$ on a $10 \mathrm{~cm}$ VAS. Participants with a $7 \mathrm{~cm}$ VAS score (VAS-7) at a pressure lower than $20 \mathrm{kPa}$ (5 HCs and $16 \mathrm{CWP}$ patients) were given a cuff pressure stimulation with a pressure $3 \mathrm{kPa}$ lower than the VAS-7 pressure during the second pain session in order not to evoke intolerable pain. The cuff was applied to the lower dominant leg, and inflation and deflation was instantaneous by a cuff algometer (NociTech). Only the resting state sessions (prior- and post-pain) and not the task-fMRI data from the pain sessions in-between were analyzed for the purpose of this study.

\section{Data analysis and statistics}

The clinical measurement parameters are presented as mean and SD. Correlation results for group with the clinical measures covariates (pain intensity, pain sensitivity, and psychological strain) were calculated, tested for significance at a Bonferroni-corrected threshold of $\alpha=0.05$ / $19=0.003$ to correct for 19 correlations, and reported with their effect size as Cohen's d.

Preprocessing of the rs-fMRI data was done with the Functional Connectivity Toolbox (CONN) version $15 \mathrm{~g}$ for MATLAB (Mathworks, Inc., Natick, MA, USA) ${ }^{45}$ using SPM12 functions (Wellcome Trust Centre for Neuroimaging, London, UK). Preprocessing consisted of rs-fMRI data realignment, coregistration to individual anatomical data, segmentation of white and grey matter and cerebrospinal fluid, normalization to a standard brain template in the Montreal Neurological Institute (MNI) coordinate space and smoothing by an 8 -mm full-width at half 


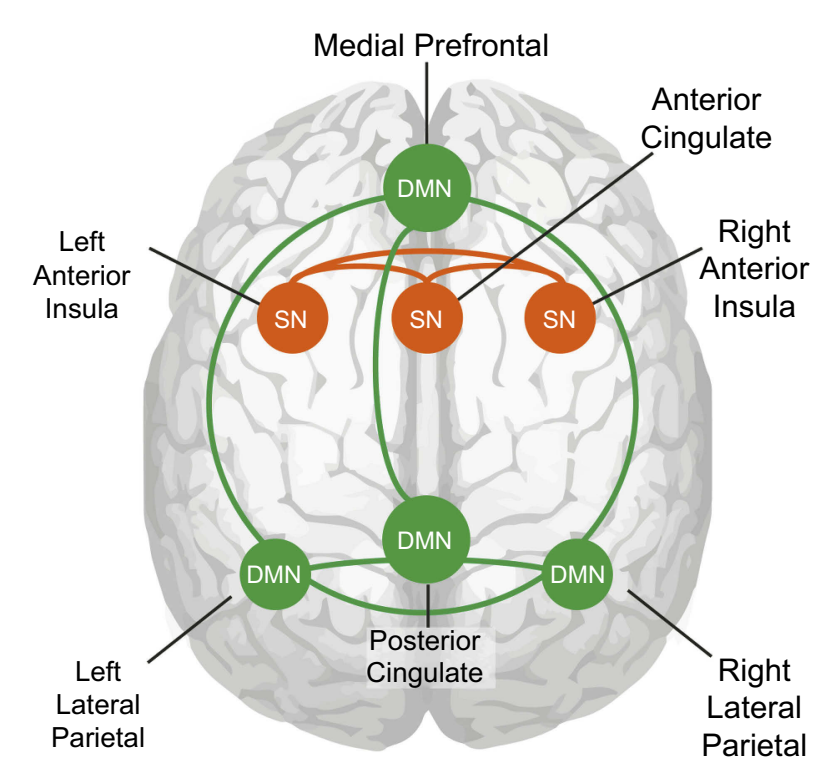

Figure I Node connections of the default mode network and the salience network. Cartoon of node connections used in this study as seen on a see-through brain. Green shows the connections between the nodes of the DMN and orange the connection between the nodes of the SN.

Abbreviations: DMN, default mode network; SN, salience network.

maximum Gaussian kernel. From the individual datasets, confounding effects defined as motion regressors (calculated during realignment), scrubbing parameters to filter out excessive motion spikes (calculated with the ARTplugin in CONN with conservative settings aiming to remove $5 \%$ of normative data), white matter, cerebrospinal fluid, and a term for linear detrending were regressed out, and the data was band-pass filtered $(0.008-0.09 \mathrm{~Hz})$.

For hypothesis (1), the main effect of group was investigated with the use of independent component analysis (ICA) in the MATLAB-based GIFT toolbox version 3.0a (Medical Image Analysis Lab, http://mia lab.mrn.org/software/gift), which enabled connectivity investigation in the whole DMN or SN based on groupderived neural network connectivity maps. For hypothesis (2), we investigated the functional connectivity changes resulting from the interaction of group with the variables of interest available for both CWP and HC (pain sensitivity, psychological strain). Furthermore, we investigated the correlation of clinical pain intensity with functional connectivity measures for the CWP group specifically. These covariate analyses were performed with region of interest (ROI)-to-ROI analysis in the CONN toolbox, which enables the investigation of connectivity changes between specific predefined ROIs. For hypothesis (3), again a ROI-to-ROI analysis was used to investigate the interaction of group with condition (baseline and post-experimental pain) in relation to the included covariates.

For the purposes of this study, the DMN was defined as a network containing the PCC, left LPC, right LPC, and MPFC. ${ }^{18,46}$ The SN was defined as a network containing the ACC, left AIC and right AIC. ${ }^{22} \mathrm{DMN}$ and SN templates that contained these regions were taken from the published template data from Laird and colleagues (component 4 for $\mathrm{SN}$, component 13 for DMN). ${ }^{46}$ These templates were then used to select DMN and SN components from the group ICA on our data from all participants. Furthermore, ROIs were created by extracting the four DMN nodes (PCC, left LPC, right LPC, MFPC) and three $\mathrm{SN}$ nodes (ACC, left AIC, right AIC) from the DMN and SN templates, with an applied threshold of z $>5$ (schematic overview of network nodes in Figure 1).

\section{Main effect of group}

A group ICA algorithm was performed in GIFT with the use of default settings including subject-specific principal component analysis for data reduction and the calculation of 25 maximally spatially independent components with an infomax algorithm. Stability of ICA findings were ensured by rerunning ICA 100 times with the use of ICASSO; the best stable run estimates were used to backreconstruct components for each participant. The mean component maps for all participants were then spatially mapped to the predefined DMN and SN templates ${ }^{46}$ to select the two components best matching each network. Within each component, the connectivity differences between groups were investigated with a correlation analysis in SPM12. The initial significance threshold was set at $P<0.001$ uncorrected to select voxels for a small-volume investigation that tested only within the DMN and $\mathrm{SN}$ template regions (as a combined mask), and tested for significance with a threshold of $P<0.05$ corrected for family wise error rate (FWE) with the use of small-volume correction.

\section{ROI-to-ROI connectivity analysis of clinical measures}

To determine functional connectivity values for each individual within our seven predefined ROIs defined as DMN and SN nodes, bivariate correlations were calculated between time signals for each combination of two ROIs. To establish that the choice of ROIs for nodes in the DMN and $\mathrm{SN}$ was consistent with literature findings on strong connectivity within the DMN and within the SN as well as negative connectivity between the DMN and $\mathrm{SN}$, the 
connectivity of the DMN PCC seed with all other nodes was investigated with a significance threshold of $P<0.001$ false discovery rate (FDR) corrected for multiple measurements over all six target nodes.

The individual bivariate correlation values were entered in correlation and linear regression ROI-toROI analyses for hypothesis testing. For these ROI-toROI functional connectivity analyses, the PCC node of the DMN and the left and right AIC nodes of the SN were selected as seed nodes in separate analyses, with the remaining six DMN and SN nodes as target nodes. Clinical pain intensity was only measured for CWP and thus only tested within the CWP group with a correlation analysis at baseline and a regression analysis to investigate the interaction of pain intensity with post-pain functional connections compared to baseline. The other two covariates; pain sensitivity and psychological strain, were tested with separate regression analyses that compared the slopes of each covariate for between-group differences related to the ROI-to-ROI functional connections at baseline or the interaction of group and post-pain functional connectivity compared to baseline.

The significance threshold for seed node analysis was set at $p<0.05$ FDR-corrected for multiple measurements (six targets), an additional Bonferroni correction of 3 was applied to correct for tests for each of the three seed ROIs used. To remove any confounding effect of age, we modeled age as a nuisance variable in all covariate analyses. Significant results that emerged are presented with their $t$ - or $\mathrm{R}^{2}$-values. This resulted in total in 6 separate two-tailed $t$-tests to test all between- and within-group covariate effects of hypotheses (2) and (3). Effect sizes are reported as Cohen's $d$ for the correlation analysis and Cohen's $f 2$ for the multivariate linear regression analyses.

\section{Results}

In line with expectations, group differences between CWP and $\mathrm{HC}$ were observed in relation to pain intensity as a huge effect $(p<0.001$, Cohen's $d=2.99)$, psychological variables as a very large effect $(p<0.001$, Cohen's $\mathrm{d}=1.49)$, PTT as a medium to large effect $(p=0.001$, Cohen's $\mathrm{d}=0.78$ ), and PDT as a medium effect ( $p=0.021$, Cohen's $d=0.55$ (Table 1). No significant differences in age, anthropometric variables or blood pressures were found.

\section{Baseline DMN and SN connectivity Whole group baseline connectivity in DMN and $\mathrm{SN}$}

High baseline connectivity for the whole participant population was found within the DMN $(t(73)=42.2$, $p<0.001$ FDR-corrected); and $\mathrm{SN}(t(73)=33.1, p<0.001$ FDR-corrected). For confirmation of expected DMN-SN negative connectivity, we observed the connectivity correlation of the PCC node of the DMN with all other nodes in all participants. Connectivity in the PCC node correlated positively to connectivity in all DMN nodes and negatively to connectivity in all SN nodes $(p<0.001$ FDR-corrected for all target nodes).

\section{Between-group connectivity differences in PCC and AIC}

At baseline, within the independent component for the DMN, the $\mathrm{HC}$ group showed stronger connectivity than the CWP group in the inferior PCC $[10-564] \mathrm{T}=5.02, p=0.04$ FWE-corrected (Figure 2). For the SN component, the CWP group had stronger connectivity than the HC group in the left AIC/superior temporal gyrus [ $\left.\begin{array}{lll}-44 & 10 & -18\end{array}\right] \mathrm{T}=5.27$, $p=0.016$ FWE-corrected (Figure 3).

\section{Effects of clinical measurements}

No effect of pain intensity on baseline connectivity

Clinical pain intensity measured as VAS pain intensityglobal was not found to correlate with altered functional connectivity related to DMN or SN nodes in the CWP group.

\section{Effect of pain sensitivity on baseline connectivity}

A comparison of pain sensitivity measured as the PTT between CWP and in HC showed that higher pain sensitivity in CWP predicted significantly increased connectivity between left and right AIC nodes of the SN as a small-tomedium effect, and increased connectivity between right AIC of the SN and left LPC of the DMN as a medium effect (Table 2).

\section{No effect of psychological strain on baseline connectivity}

There were no significant between- or within-group effects observed of functional connectivity related to DMN or SN nodes correlating with higher psychological strain.

\section{No post-experimental pain effects}

No between-group effects of post-experimental pain compared to baseline were found. No regression effects for pain sensitivity or psychological strain were found for the 


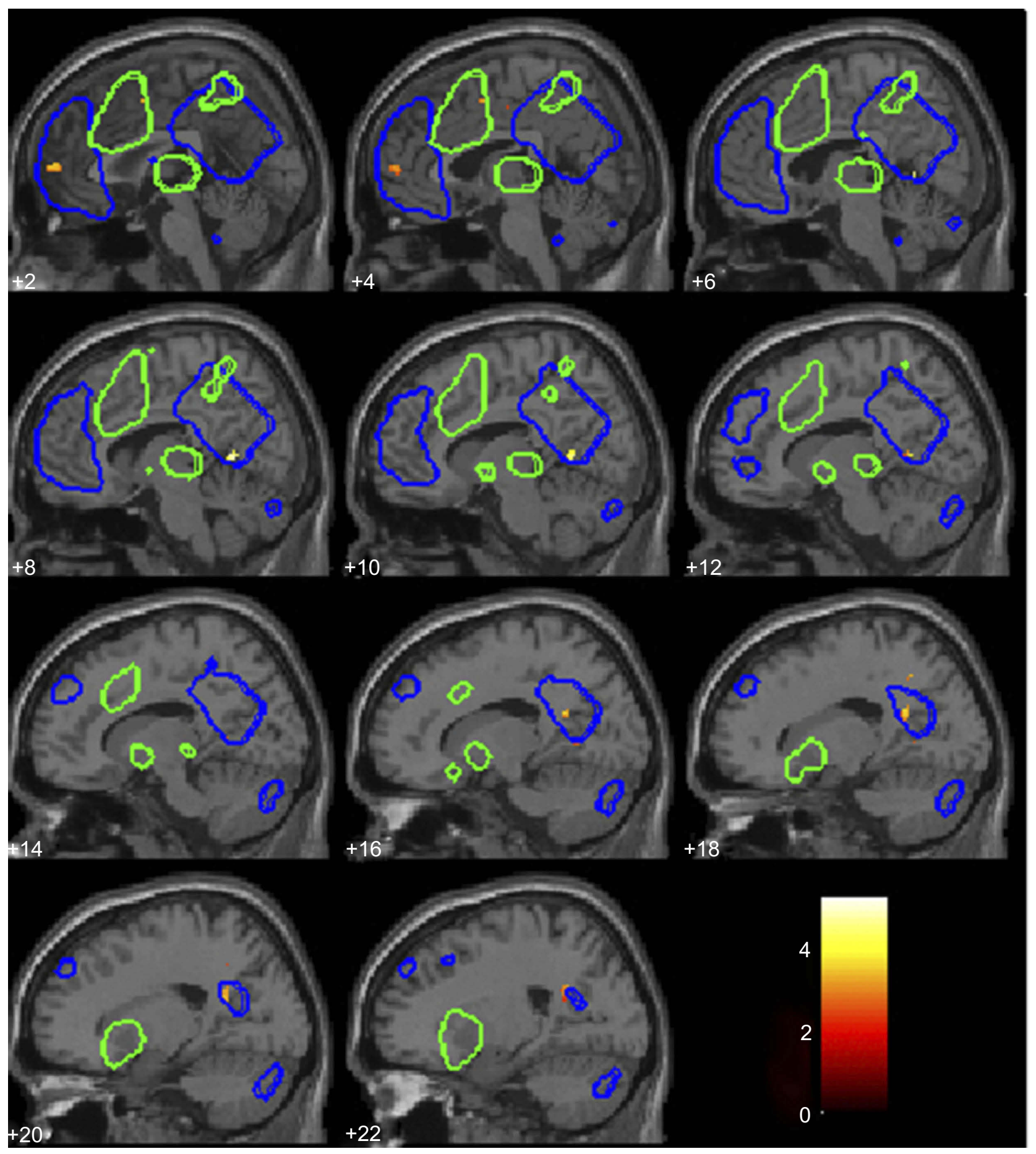

Figure 2 Between-group difference within the default mode network independent component. Connectivity differences for HCs compared to CWP. The blue overlay shows the DMN template, the green overlay shows the SN template, these two templates together were used for small-volume-correction and results were significant at $p<0.05 \mathrm{FWE}$-corrected. For illustrative purposes, in this figure the connectivity result map was thresholded at $p<0.0 \mathrm{I}$ uncorrected ( $t$-value is shown in the legend insert), not masked by the templates. The numbers denote the $\mathrm{x}$-coordinate, significant difference in connectivity was found at $\mathrm{x}=10$ and adjacent slides in the PCC.

Abbreviations: HC, healthy control; CWP, chronic widespread pain; DMN, default mode network, SN, salience network; FWE, family-wise error rate; PCC, posterior cingulate cortex.

group $\mathrm{x}$ condition interactions post-experimental pain compared to baseline. No signficant effects were found post-experimental pain within the CWP group for the CWP-only pain intensity covariate.

\section{Discussion}

The CWP group differed significantly from the HC group and showed decreased connectivity in the inferior PCC in the DMN network and increased connectivity in the left 


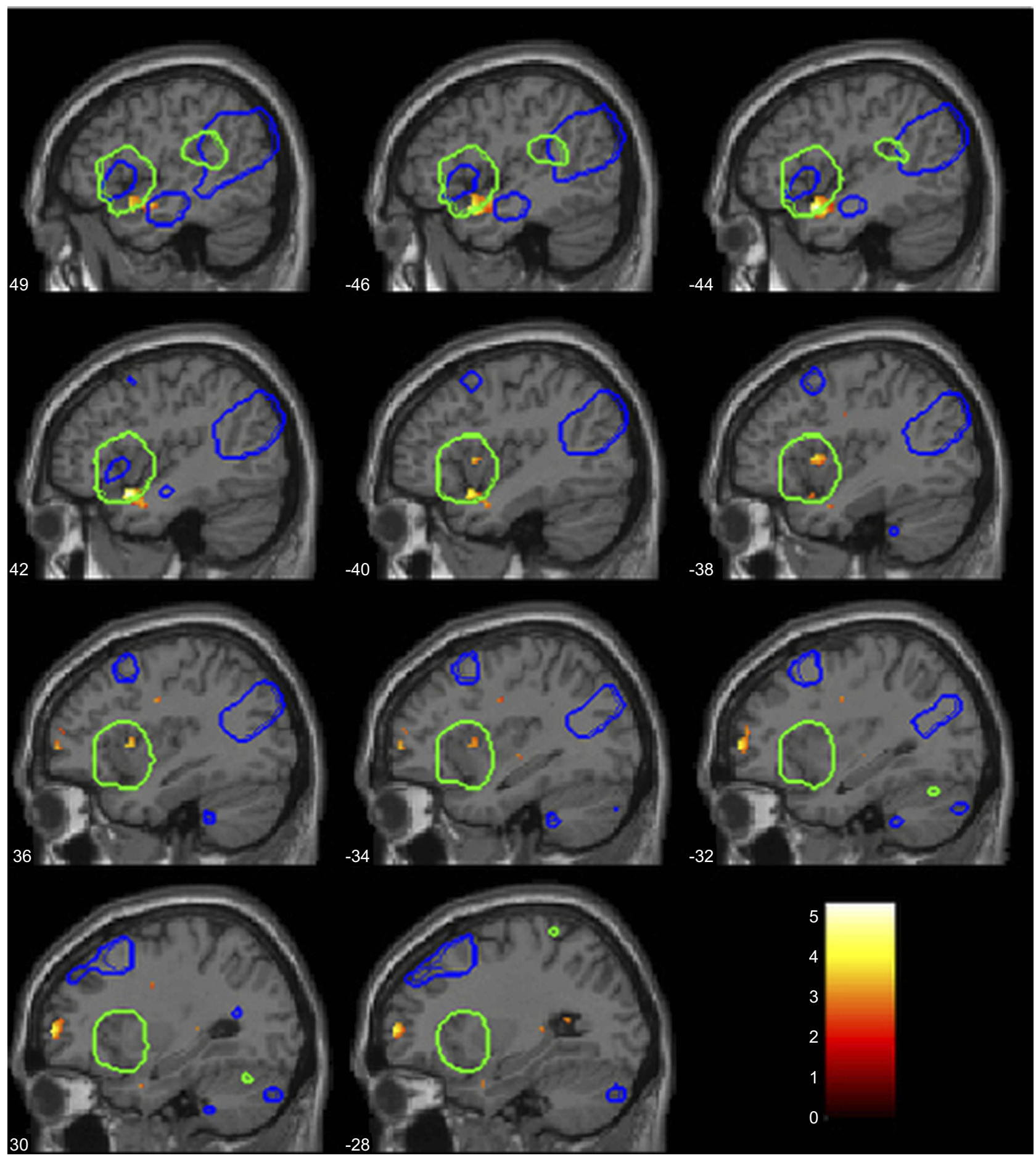

Figure 3 Between-group difference within the salience network independent component. Connectivity differences for CWP compared to HCs. The blue overlay shows the DMN template, the green overlay shows the SN template, these two templates together were used for small-volume-correction and results were significant at $p<0.05 \mathrm{FWE}-$ corrected. For illustrative purposes, in this figure the connectivity result map was thresholded at $p<0.0 \mathrm{I}$ uncorrected ( $t$-value is shown in the legend insert), not masked by the templates. The numbers denote the $\mathrm{x}$-coordinate, significant difference in connectivity was found at $\mathrm{x}=-44$ and adjacent slides in the left anterior insula/superior temporal gyrus.

Abbreviations: CWP, chronic widespread pain; HC, healthy control; DMN, default mode network, SN, salience network; FWE, family-wise error rate.

AIC in the SN network. This supported our first hypothesis on affected DMN and SN connectivity in CWP. Furthermore, functional connectivity differences between CWP and HC proved to be dependent on pain sensitivity as stated in our second hypothesis. However, there were no differences observed between CWP and HC in functional connectivity related to DMN or SN nodes as an effect of psychological strain, nor in the CWP group as an effect of 
Table 2 Regression analysis for baseline rs-fMRI comparison between the CWP group and the HC group on the covariates PTT as a measure of pain sensitivity, and the construct psychological strain

\begin{tabular}{|c|c|c|c|c|c|c|}
\hline Covariate & Seed-ROI & Target-ROI & $t$-value $(d f=70)$ & $\mathbf{R}^{2}$ & Effect size (Cohen's $f 2$ ) & $p$-value \\
\hline \multicolumn{7}{|c|}{ Baseline resting state } \\
\hline $\begin{array}{l}\text { PTT } \\
\text { Psychological strain }\end{array}$ & $\begin{array}{l}\text { SN RAIC } \\
\text { SN RAIC } \\
\text { SN LAIC } \\
\text { DMN PCC }\end{array}$ & $\begin{array}{l}\text { DMN LPC } \\
\text { SN LAIC } \\
\text { SN RAIC }\end{array}$ & $\begin{array}{l}3.31 \\
2.92 \\
2.92\end{array}$ & $\begin{array}{l}0.15 \\
0.10 \\
\text { n.s. } \\
\text { n.s. } \\
\text { n.s. }\end{array}$ & $\begin{array}{l}0.176 \\
0.111\end{array}$ & $\begin{array}{l}0.026 \\
0.043 \\
0.085 \\
>0.1 \\
>0.1\end{array}$ \\
\hline \multicolumn{7}{|c|}{ Post-pain versus baseline resting state } \\
\hline $\begin{array}{l}\text { PTT } \\
\text { Psychological strain }\end{array}$ & & & & $\begin{array}{l}\text { n.s. } \\
\text { n.s. }\end{array}$ & & $\begin{array}{l}>0.1 \\
>0.1\end{array}$ \\
\hline
\end{tabular}

Notes: The $p$-value is FDR -corrected for seven targets, subsequently Bonferroni-corrected for three seeds. Target ROls and $t$-values of results with $p$-value below 0.1 are shown.

Abbreviations: rs-fMRI, resting state functional magnetic resonance imaging; CWP, chronic widespread pain; HC, healthy control; PTT, pain tolerance threshold; ROI, region of interest; df, degrees of freedom; SN, salience network; RAIC, right anterior insular cortex; LAIC, left anterior insular cortex; DMN, default mode network; LPC, lateral parietal cortex; PCC, posterior cingulate cortex; n.s., not significant.

clinical pain intensity. Neither did functional connectivity after experimental pain show significant changes from observed baseline connectivity, thus no support for our third hypothesis was found. The results are obtained from an all-female study population, which enables better comparison to literature on $\mathrm{FM}-\mathrm{a}$ female-dominated diagnosis.

The decreased connectivity within the DMN has previously been observed in studies on chronic non-widespread pain. ${ }^{11,12}$ There are a few studies that identified the PCC in the DMN as a differentiating region of altered functional connectivity between controls and patients. ${ }^{11,47}$ However, decreased DMN connectivity is not in concordance with the finding of FM patients exhibiting upregulation of DMN connectivity. ${ }^{8}$ The AIC has been observed in several studies to have increased connectivity with the DMN. Increased connectivity within the SN regardless of covariates in CWP or FM has not often been reported, but increased correlation of the AIC with ACC as well as the hippocampus has been shown as an effect of acute pressure pain in $\mathrm{FM}^{10}$ That study reports AIC ROIs which have coordinates overlapping with our significant cluster within the AIC of the SN, however, it can be observed that this significant cluster is anatomically located in the superior temporal gyrus (Figure 3, at x-coordinate -44 ). ${ }^{10}$ Our finding of betweengroup differences in the $\mathrm{SN}$ without accounting for applied pressure pain is to the best of our knowledge a novel finding. Interestingly, the visible but not significant cluster that is located anatomically in the left AIC (Figure 3, at X-coordinate -36) was overlapping with the AIC ROI from the
2014 study of Ichesco et al that found significant betweengroup differences related to left AIC unrelated to any covariate. $^{48}$ Our results could indicate that the CWP patients are dissimilar from FM patients, and have different connectivity changes in the left AIC. However, since the literature on FM is sparse and not highly consistent, our results in combination with the literature might suggest that there are multiple regions in the left AIC/superior temporal gyrus that contribute to chronic widespread pain characteristics, in a fluid rather than consistent fashion. We may even speculate that if the left AIC/superior temporal cluster is modulated by acute pain, any pain caused by the discomfort of an fMRI scanning session may have been affecting the CWP patients more than the HCs. We believe that our findings of between-group differences contribute to the scarce literature on CWP, and gives insight of connectivity changes in the whole DMN and SN in contrast to other studies that present findings within small ROIs.

\section{Salience network disruption correlates to higher pain sensitivity}

In the current study, higher pain sensitivity but not higher pain intensity was associated with increased connectivity between DMN and SN nodes as well as with increased within-network SN connectivity for CWP compared to HC at baseline. Pain sensitivity in this study was measured as the tolerance threshold for pain. In the study of Napadow et al on FM where a network analysis was obtained with an ICA, the authors showed an association of increased 
connectivity between DMN and insula to higher pain intensity. ${ }^{8}$ Baliki et al showed a strong correlation between pain intensity and connectivity between the MPFC and insula that reproduced over different chronic regional pain diagnoses. ${ }^{11}$ Furthermore, ankylosing spondylitis patients showed increased connectivity between the DMN and $\mathrm{SN}$, mainly driven by a node in the temporoparietal junction, in relation to pain intensity. ${ }^{13}$ This region has not been included in our SN investigations as it is not primarily implied in chronic pain connectivity changes. ${ }^{5}$ That pain intensity does not evoke a similar effect as pain sensitivity in the current study may reflect the diffuse nature of CWP conditions, which is especially in FM characterized by varying painful sites all over the body in contrast to localized intense pain.

It has been suggested that a disturbed $\mathrm{SN}$ is the cause of increased connectivity between the DMN and SN. ${ }^{13}$ It is plausible that observed $\mathrm{SN}$ disturbance by chronic pain may be an effect of structural changes in the $\mathrm{SN}$, such as observed as volume decrease of the ACC and AIC in FM. $^{49}$ In conclusion, this new finding of interaction of pain sensitivity with SN and DMN-SN functional connectivity in CWP further strengthens the theory of SN disruption in chronic pain patients by the continuous input of salient stimuli containing nociceptive information. ${ }^{13}$ This study shows a differentiation between the influence of pain sensitivity and pain intensity, advocating the use of pain sensitivity measurements as a modulating factor in network connectivity studies.

\section{Absence of psychological strain or experimental pain effects}

The psychological strain variable in this study included scales reflecting on depression, anxiety, and pain catastrophizing. The high internal consistency of the construct together with its observed correlation to connectivity changes advocates the useability of employing this construct for psychological strain. Many sub-aspects of major depression have been associated with increased DMN connectivity, however previous findings are not highly consistent. $^{30}$ In contrast, higher trait anxiety has been associated with reduced DMN connectivity. ${ }^{27}$ This study, however, found no evidence for a distinctive effect of psychological strain on connectivity related to DMN and SN nodes. Therefore, we cannot conclude that general psychological strain is a cause or an effect of DMN or $\mathrm{SN}$ alterations. Indeed it is possible that depressive symptoms may increase DMN connectivity in CWP patients while anxiety symptoms decrease this connectivity, which would then not be observed as a clear effect on DMN connectivity for the construct psychological strain. However, when we freely explored our data, we did not find clear effects linked to just the depression or anxiety measures. Thus, there might be an absence of consistent correlation of psychological strain aspects in CWP with DMN and SN connectivity. This may lead to a shift of focus towards other neural networks in order to better understand changed neural aspects of CWP in relation to psychological strain.

Existing literature on connectivity alterations as effect of acute or experimental pain in chronic pain patients is conflicting and sparse. Applied pressure pain has been shown to increase connectivity of the ACC with the hippocampus which is part of the descending pain modulatory system in FM patients. ${ }^{50}$ Chronic lower back pain patients with an increased clinical pain caused by physical maneuvering have exhibited increased connectivity between the DMN and mid-insula - a region adjacent to the AIC node of $\mathrm{SN}$ and involved in affective pain processing. ${ }^{33}$ In contrast, the DMN showed decreased connectivity with the pregenual ACC, adjacent to the ACC associated with the $\mathrm{SN}^{33}$ A study that investigated functional connectivity after a physical exercise intervention aimed at improvement of symptoms in FM showed to affect insular connections with the sensorimotor cortex. ${ }^{51}$ The authors suggested that a restoration of functional connectivity through physical exercise could have been established. ${ }^{51}$ However, our results do not indicate an altering effect of experimental pain on functional connecitvity differences within- or between-groups in relation to either of the covariates. We may tentatively conclude that any effects previously observed between the DMN, insula and ACC are likely either not associated with the anterior part of the insula or very short-lived and regulated back to normal connectivity in CWP after several minutes.

\section{Limitations}

Whereas the current study population was larger than most studies on CWP and FM, it still is a limited sample size for more complex analyses, therefore we could not control factors such as smoking, alcohol, or exercise in the CWP group. The group size and heterogeneity also limited the separate analyses that could be included; it would be informative to investigate in future studies whether different types of psychological strain and the different 
questionnaires would have differentiating results. The choice for a compound variable was based on the relatively low scores on several scales included in the psychological strain constructs. Also in this case, future research is needed to understand whether anxiety symptoms have a modulatory role in decreased DMN connectivity in CWP, and moreover, whether other intrinsic neural networks such as those regulating executive control functions may be affected, as executive control networks have been implied in chronic pain conditions in some literature. ${ }^{27,30}$

We have used a VAS to measure pain intensity as well as pain sensitivity because this measure is quickly applied, sensitive to pain experience, and widely used and therefore more easily comparable over studies. ${ }^{52}$ The limitations of using a VAS are the confounding effects of inconsistent interpretation of scale by the participants as well as possible ceiling effects in patients with high pain. Healthy partipants tested for pain sensitivity may report stimulus intensity rather than pain for low-pain trials, and therefore the measurement may lose sensitivity. ${ }^{53}$ Follow-up studies would therefore preferably include several pain measures to validate findings.

Most patients were on different types of prescribed medication. The patients were asked to refrain from pain medication during the experiment day, but due to both ethical aspects and practical issues it was not possible to investigate these patients taken off their medication for several days/weeks. It is likely that medication intake affects functional connectivity, ${ }^{11}$ and because of the limited wash-out period medication has to be regarded as a possible confounding factor of the presented results. However, even though the current study cannot model CWP functional connectivity alterations fully, it can contribute to the currenty incomplete understanding of differences between CWP patients and HCs, and of the contribution of pain sensitivity to the differences between groups.

\section{Conclusion}

The results of this study demonstrate functional connectivity changes in the DMN and SN for CWP patients and an effect of pain sensitivity measured as pain tolerance threshold on SN and SN-to-DMN connectivity. Pain sensitivity is related to increased connectivity between left and right $\mathrm{AIC}$ as well as to increased connectivity between the right AIC and the left LPC in CWP. Pain intensity nor psychological strain showed any correlations with functional connectivity changes in either the DMN or SN.
Also, no effect of experimental pain was observed. This study contributes to the understanding of DMN and SN alterations in CWP, and implies that increased connectivity between these networks is modulated by pain sensitivity.

\section{Acknowledgments}

This work was supported by a research grant from Forskningsrådet i Sydöstra Sverige (FORSS [Medical Research Council of Southeast Sweden]) and the Swedish Research Council. The Center for Neuroplasticity and Pain (CNAP) is funded by the Danish National Research Foundation (DNRF121). The authors would like to thank Eva-Britt Lind for assisting with data collection, and all participants for their cooperation.

\section{Disclosure}

Dr. Helene van Ettinger-Veenstra and Prof. Dr. Björn Gerdle report grants from FORSS and from the Swedish Research Council during the conduct of the study. The authors report no other conflicts of interest in this work.

\section{References}

1. Grimby-Ekman A, Gerdle B, Björk J, Larsson B. Comorbidities, intensity, frequency and duration of pain, daily functioning and health care seeking in local, regional, and widespread pain - a descriptive population-based survey (SwePain). BMC Musculoskelet Disord. 2015;16:165. doi:10.1186/s12891-015-0631-1

2. Sluka KA, Clauw DJ. Neurobiology of fibromyalgia and chronic widespread pain. Neuroscience. 2016;338:114-129. doi:10.1016/j. neuroscience.2016.06.006

3. Butler S, Landmark T, Glette M, Borchgrevink P, Woodhouse A. Chronic widespread pain - the need for a standard definition. Pain. 2015;157(3):1. doi:10.1097/j.pain.0000000000000417

4. Cagnie B, Coppieters I, Denecker S, Six J, Danneels L, Meeus M. Central sensitization in fibromyalgia? A systematic review on structural and functional brain MRI. Semin Arthritis Rheum. 2014;44 (1):68-75. doi:10.1016/j.semarthrit.2014.01.001

5. Coppieters I, Meeus M, Kregel J, et al. Relations between brain alterations and clinical pain measures in chronic musculoskeletal pain: a systematic review. J Pain. 2016;17(9):949-962. doi:10.1016/ j.jpain.2016.04.005

6. Apkarian AV, Bushnell MC, Treede R-D, Zubieta J-K. Human brain mechanisms of pain perception and regulation in health and disease. Eur J Pain. 2005;9(4):463-484. doi:10.1016/j.ejpain.2004.11.001

7. Jensen KB, Regenbogen C, Ohse MC, Frasnelli J, Freiherr J, Lundström JN. Brain activations during pain: a neuroimaging meta-analysis of pain patients and healthy controls. Pain. 2016;157 (6):1279-1286. doi:10.1097/j.pain.0000000000000517

8. Napadow V, LaCount L, Park K, As-Sanie S, Clauw DJ, Harris RE. Intrinsic brain connectivity in fibromyalgia is associated with chronic pain intensity. Arthritis Rheum. 2010;62(8):2545-2555. doi:10.1002/ art. 27497

9. Napadow V, Kim J, Clauw DJ, Harris RE. Decreased intrinsic brain connectivity is associated with reduced clinical pain in fibromyalgia. Arthritis Rheum. 2012;64(7):2398-2403. doi:10.1002/art.34412 
10. Ichesco E, Puiu T, Hampson JP, et al. Altered fMRI resting-state connectivity in individuals with fibromyalgia on acute pain stimulation. Eur J Pain. 2016;20(7):1079-1089. doi:10.1002/ejp.832

11. Baliki MN, Mansour AR, Baria AT, Apkarian AV. Functional reorganization of the default mode network across chronic pain conditions. PLoS One. 2014;9(9):e106133. doi:10.1371/journal.pone.0106133.

12. Kregel J, Meeus M, Malfliet A, et al. Structural and functional brain abnormalities in chronic low back pain: a systematic review. Semin Arthritis Rheum. 2015;45(2):229-237. doi:10.1016/ j.semarthrit.2015.05.002

13. Hemington KS, Wu Q, Kucyi A, Inman RD, Davis KD. Abnormal cross-network functional connectivity in chronic pain and its association with clinical symptoms. Brain Struct Funct. 2016;221 (8):4203-4219. doi:10.1007/s00429-015-1161-1

14. Fox MD, Snyder AZ, Vincent JL, Corbetta M, Van Essen DC, Raichle ME. The human brain is intrinsically organized into dynamic, anticorrelated functional networks. Proc Natl Acad Sci U S A. 2005;102(27):9673-9678. doi:10.1073/pnas.0504136102

15. Smith SM, Fox PMTM, Miller KL, et al. Correspondence of the brain's functional architecture during activation and rest. Proc Natl Acad Sci U S A. 2009;106(31):13040-13045. doi:10.1073/ pnas. 0905267106

16. Otti A, Guendel H, Läer L, et al. I know the pain you feel—how the human brain's default mode predicts our resonance to another's suffering. Neuroscience. 2010;169(1):143-148. doi:10.1016/j. neuroscience.2010.04.072

17. Kucyi A, Salomons TV, Davis KD. Mind wandering away from pain dynamically engages antinociceptive and default mode brain networks. Proc Natl Acad Sci. 2013;110(46):18692-18697. doi:10.1073/pnas. 1312902110

18. Fox MD, Raichle ME. Spontaneous fluctuations in brain activity observed with functional magnetic resonance imaging. Nat Rev Neurosci. 2007;8(9):700-711. doi:10.1038/nrn2201

19. Tracey I, Mantyh PW. The cerebral signature for pain perception and its modulation. Neuron. 2007;55(3):377-391. doi:10.1016/j. neuron.2007.07.012

20. Seeley WW, Menon V, Schatzberg AF, et al. Dissociable intrinsic connectivity networks for salience processing and executive control. $J$ Neurosci. 2007;27(9):2349-2356. doi:10.1523/JNEUROSCI.558706.2007

21. Wager TD, Atlas LY, Lindquist MA, Roy M, Woo C-W, Kross E. An fMRI-based neurologic signature of physical pain. $N$ Engl $J$ Med. 2013;368(15):1388-1397. doi:10.1056/NEJMoa1204471

22. Menon V. Salience network. In Toga, A.W. (Ed.), Brain Mapping: An Encyclopedic Reference, Vol. 2. Academic Press, Elsevier, 2015:597-611. doi:10.1016/B978-0-12-397025-1.00052-X

23. Di X, Biswal BB. Dynamic brain functional connectivity modulated by resting-state networks. Brain Struct Funct. 2015;220(1):37-46. doi:10.1007/s00429-013-0634-3

24. Peolsson M, Börsbo B, Gerdle B. Generalized pain is associated with more negative consequences than local or regional pain: a study of chronic whiplash-associated disorders. J Rehabil Med. 2007;39 (3):260-268. doi:10.2340/16501977-0052

25. Börsbo B, Gerdle B, Peolsson M. Impact of the interaction between self-efficacy, symptoms and catastrophising on disability, quality of life and health in with chronic pain patients. Disabil Rehabil. 2010;32 (17):1387-1396. doi:10.3109/09638280903419269

26. Börsbo B, Peolsson M, Gerdle B. The complex interplay between pain intensity, depression, anxiety and catastrophising with respect to quality of life and disability. Disabil Rehabil. 2009;31 (19):1605-1613. doi:10.1080/09638280903110079

27. Modi S, Kumar M, Kumar P, Khushu S. Aberrant functional connectivity of resting state networks associated with trait anxiety. Psychiatry Res Neuroimaging. 2015;234(1):25-34. doi:10.1016/j. pscychresns.2015.07.006
28. Peterson A, Thome J, Frewen P, Lanius RA. Resting-state neuroimaging studies: a new way of identifying differences and similarities among the anxiety disorders? Can J Psychiatry. 2014;59(6):294-300. doi: $10.1177 / 070674371405900602$

29. Pannekoek JN, van der Werff SJA, van Tol MJ, et al. Investigating distinct and common abnormalities of resting-state functional connectivity in depression, anxiety, and their comorbid states. Eur Neuropsychopharmacol. 2015;25(11):1933-1942. doi:10.1016/j. euroneuro.2015.08.002

30. Brakowski J, Spinelli S, Dörig N, et al. Resting state brain network function in major depression - depression symptomatology, antidepressant treatment effects, future research. $J$ Psychiatr Res. 2017;92:147-159. doi:10.1016/j.jpsychires.2017.04.007

31. Borsook D, Sava S, Becerra L. The pain imaging revolution - advancing pain into the 21st century. Neuroscientist. 2010;16(2):171-185. doi: $10.1177 / 1073858409349902$. The

32. Zhang S, Wu W, Huang G, et al. Resting-state connectivity in the default mode network and insula during experimental low back pain. Neural Regen Res. 2014;9(2):135-142. doi:10.4103/16735374.125341

33. Loggia ML, Kim J, Gollub RL, et al. Default mode network connectivity encodes clinical pain: an arterial spin labeling study. Pain. 2013;154(1):24-33. doi:10.1016/j.pain.2012.07.029

34. Kucyi A, Davis KD. The dynamic pain connectome. Trends Neurosci. 2015;38(2):86-95. doi:10.1016/j.tins.2014.11.006

35. Wolfe F, Smythe HA, Yunus MB, et al. The American College of Rheumatology 1990 criteria for the classification of fibromyalgia. Arthritis Rheum. 1990;33(2):160-172. doi:10.1002/art.1780330203

36. Zigmond AS, Snaith RP. The hospital anxiety and depression scale. Acta Psychiatr Scand. 1983;67(6):361-370.

37. Beck AT, Ward CH, Mendelson M, Mock J, Erbaugh J. An inventory for measuring depression. Arch Gen Psychiatry. 1961;4(6):561-571. doi:10.1001/archpsyc.1961.01710120031004

38. Beck AT, Epstein N, Brown G, Steer RA. An inventory for measuring clinical anxiety: psychometric properties. J Consult Clin Psychol. 1988;56(6):893-897. doi:10.1037/0022-006X.56.6.893

39. Reiss S, Peterson RA, Gursky DM, McNally RJ. Anxiety sensitivity, anxiety frequency and the prediction of fearfulness. Behav Res Ther. 1986;24(1):1-8. doi:10.1016/0005-7967(86)90143-9

40. McCracken LM, Dhingra L. A short version of the Pain Anxiety Symptoms Scale (PASS-20): preliminary development and validity. Pain Res Manag. 2002;7(1):45-50.

41. Sullivan MJL, Bishop SR, Pivik J. The pain catastrophizing scale: development and validation. Psychol Assess. 1995;7(4):524-532. doi:10.1037/1040-3590.7.4.524

42. Lemming D, Börsbo B, Sjörs A, et al. Cuff pressure pain detection is associated with both sex and physical activity level in nonathletic healthy subjects. Pain Med (United States). 2017;18(8):1573-1581. doi:10.1093/pm/pnw309

43. Polianskis R, Graven-Nielsen T, Arendt-Nielsen L. Spatial and temporal aspects of deep tissue pain assessed by cuff algometry. Pain. 2002;100(1-2):19-26.

44. Lemming D, Börsbo B, Sjörs A, et al. Single-point but not tonic cuff pressure pain sensitivity is associated with level of physical fitness a study of non-athletic healthy subjects. PLoS One. 2015;10(5): e0125432. doi:10.1371/journal.pone.0125432

45. Whitfield-Gabrieli S, Nieto-Castanon A. Conn: a functional connectivity toolbox for correlated and anticorrelated brain networks. Brain Connect. 2012;2(3):125-141. doi:10.1089/brain.2012.0073

46. Laird AR, Fox PM, Eickhoff SB, et al. Behavioral interpretations of intrinsic connectivity networks. $J$ Cogn Neurosci. 2011;23 (12):4022-4037. doi:10.1162/jocn_a_00077

47. Kornelsen J, Sboto-Frankenstein U, McIver T, et al. Default mode network functional connectivity altered in failed back surgery syndrome. J Pain. 2013;14(5):483-491. doi:10.1016/J.JPAIN.2012.12.018 
48. Ichesco E, Schmidt-Wilcke T, Bhavsar R, et al. Altered resting state connectivity of the insular cortex in individuals with fibromyalgia. J Pain. 2014;15(8):815-826.e1. doi:10.1016/j.jpain.2014.04.007

49. Kuchinad A, Schweinhardt P, Seminowicz DA, Wood PB, Chizh BA, Bushnell MC. Accelerated brain gray matter loss in fibromyalgia patients: premature aging of the brain? J Neurosci. 2007;27 (15):4004-4007. doi:10.1523/JNEUROSCI.0098-07.2007

50. Jensen KB, Loitoile R, Kosek E, et al. Patients with fibromyalgia display less functional connectivity in the brain's pain inhibitory network. Mol Pain. 2012;8:32. doi:10.1186/1744-8069-8-32
51. Flodin P, Martinsen S, Mannerkorpi K, et al. Normalization of aberrant resting state functional connectivity in fibromyalgia patients following a three month physical exercise therapy. NeuroImage Clin. 2015;9:134-139. doi:10.1016/j.nicl.2015.08. 004

52. Haefeli M, Elfering A. Pain assessment. Eur Spine J. 2006;15(Suppl 1):S17-S24. doi:10.1007/s00586-005-1044-x

53. Kemp J, Despres O, Dufour A. Unreliability of the visual analog scale in experimental pain assessment: a sensitivity and evoked potentials study. Pain Physician. 2012;15(5):E693-E699.

\section{Publish your work in this journal}

The Journal of Pain Research is an international, peer reviewed, open access, online journal that welcomes laboratory and clinical findings in the fields of pain research and the prevention and management of pain. Original research, reviews, symposium reports, hypothesis formation and commentaries are all considered for publication. The manuscript management system is completely online and includes a very quick and fair peer-review system, which is all easy to use. Visit http:// www.dovepress.com/testimonials.php to read real quotes from published authors. 